
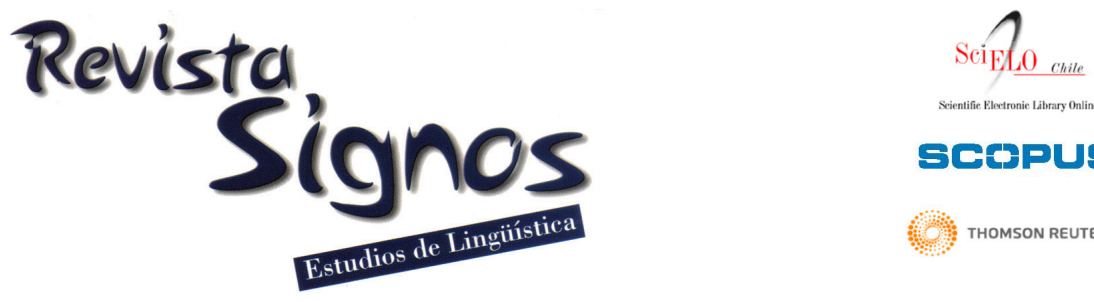

\title{
Intertextualidad a partir del establecimiento de status: Alcances sobre la relación entre contenido y superestructura en los discursos de juicios orales*
}

Intertextuality from the establishment of status: Remarks on the relation between content and superestructure in the discourse of oral trials

\author{
Cristián Noemi \\ cnoemi@userena.cl \\ Universidad de La Serena \\ Chile
}

Resumen: A partir de un método cualitativo que sigue los procedimientos establecidos por la grounded theory, el trabajo procura determinar el rol que cumple la categoría retórica clásica de status en el entramado intertextual del discurso de los juicios orales. Sobre la base de la evidencia obtenida en un corpus de 25 juicios, se muestra que la noción semántica de status cumple un papel en el sentido de generar un proceso textual que pauta la conformación superestructural de este tipo de discursos, por lo que se avanza respecto del conocimiento sobre la relación existente entre contenido y forma.

Palabras Clave:Argumentación, juicio oral, status, superestructura. 


\begin{abstract}
Using a qualitative method based on the procedures established by the grounded theory, this paper tries to determine the role that fulfills the rhetorical classic category of status in the intertextual network of the discourse of the oral trials. On the basis of the evidence obtained in a corpus of 25 oral trials, the paper shows that the semantic notion of status plays a role in terms of generating a textual process that leads the superstructural conformation of this kind of discourses, shedding hereby new lights on the existing relationship between content and form.
\end{abstract}

Key Words: Argumentation, oral trials, status, superstructure.

\section{INTRODUCCIÓN}

La Región de Coquimbo, Chile, fue seleccionada a partir del año 2000 como región piloto para la aplicación de la Reforma Procesal Penal chilena, modalidad que incluyó, entre otras innovaciones, la incorporación del juicio oral como instancia resolutiva prioritaria de los procesos judiciales. De esta manera, se instauró una nueva forma de práctica social, una forma de acción entre dos personas (partes) que se articula a partir de un uso lingüístico oral contextualizado, orientado a unos propósitos definidos (sentencia favorable) y en interdependencia del contexto (normas, valores, creencias, juicios, prejuicios, ideologías, etc.).

A diferencia de otros países en los que el juicio oral constituye una práctica habitual, en Chile no existe suficiente experiencia, tanto en lo que concierne a la formación de profesionales expertos en estrategias argumentales, como en investigación de discurso argumentativo de índole legal. En términos generales, el problema ha recibido atención tanto a partir del marco doctrinario de la teoría retórica clásica (Barthes, 1974; Adams, 1983; Murphy, 1989; Aristóteles, 1990; Albaladejo, 1993; Mayoral, 1994), como por estudios realizados desde la perspectiva de la lógica formal (Toulmin, Rieke \& Janik, 1979; Rivano, 1984; Van Eemeren, Grootendorst \& Kruiger, 1987; Atienza, 1993; Walton, 1996; Campagna \& Lazzaretti, 1998), lo que ha proporcionado documentación basal relevante tanto en lo que respecta al conocimiento sintagmático del discurso jurídico como al paradigmático y al lógico, a través del análisis de estructuras formales del razonamiento. En terreno propiamente penal, la investigación precedente (Alexy, 1989; Aarnio \& MacCormick, 1992; Feteris, 1997), entre otros, ha estado abocada principalmente a establecer las correspondencias entre los mecanismos de argumentación y los marcos legales que le sirven de soporte.

La naturaleza dialéctica del juicio oral supone una objeción implícita al racionalismo propio de la lógica formal en cuanto mecanismo de prueba y asume que una supuesta 'verdad' puede emanar simplemente a partir de la discusión y contraste de pareceres (González, 1988; Martín, 1998). De este modo, su proceder tiende a neutralizar en gran medida las categorías de verdad y evidencia del método demostrativo, dando cabida a la del verosímil, propio del método argumentativo (Perelman \& Olbrechts-Tyteca, 1989), concepción de aparente quiebre con el razonamiento cartesiano que encuentra, afortunadamente, en la tradición escritural de Occidente abundante tratamiento, a partir del cuerpo doctrinario de la teoría retórica clásica (Murphy, 1989; Aristóteles, 1990; Cicerón, 1991).

En atención a las consecuencias derivadas de esta práctica discursiva, el juicio oral (al igual que otras prácticas argumentativas similares) supone para la teoría lingüística contemporánea una tarea ineludible: intentar develar el conjunto de conocimientos compartidos de que disponen los hablantes para actuar, por medio de sus posibilidades lingüísticas, en procura de unos fines determinados, en situaciones comunicativas concretas, i.e., el saber lingüístico cultural que completa el conocimiento puramente 'gramatical' de una lengua y que permite al sujeto 'instalarse' en un contexto histórico determinado mediante la construcción de enunciados verosímiles, retóricamente válidos desde una perspectiva histórica y cultural determinada (Laborda, 1996; Noemi, 1999; van Dijk, 200I). 


\section{Marco teórico}

El fenómeno de intertextualidad en el tipo de discurso de los juicios orales resulta una cuestión obvia. Al discutir respecto del fenómeno de coherencia, De Beaugrande y Dressler (198I) refieren el tema particularmente en el caso de las argumentaciones, donde el enunciador ha de tener continuamente 'en mente' tanto el texto que ha generado previamente en una relación de cotexto (Hanks, 2000), como el producido por su oponente en una relación de metatextualidad (Genette, 1992), a fin de conformar discurso contra argumentativo, $y$ en el que, por ejemplo, el receptor final (juez o mediador) requerirá acudir, en diversos tipos de relaciones intertextuales, a numerosos textos previos, como el de la fiscalía, el de la defensa, el de la norma jurídica, el de los testigos, etc., para evaluar la verosimilitud del texto presentado a consideración.

En este tenor, el tema se inscribe a partir de Kristeva (1967) en una concepción de texto concebido en términos de proceso, en su doble calidad de feno y de genotexto, que niega su clausura y sentido unilateral, esto es, intertextualidad como propiedad ontológica del objeto, lo que problematiza su estatuto epistemológico en tanto cruce de conjunción de diversos sistemas significantes, laberinto que, en opinión de Barthes (1973), no tiene más ley que el infinito de sus incrementos.

La noción de intertextualidad barthiana, códigos diversos que transitan por caminos inesperados de la vida discursiva, encuentra, a su vez, un vínculo de interés con el concepto de 'representación social' (Farr, 1988), acuñado por la psicología social, en atención a que el conjunto de lo preconfigurado (en prácticas textuales previas) permite al sujeto enunciador, echando mano de su competencia en los 'juegos de lenguaje' en sentido wittgensteiniano, elaborar (o interpretar, según el caso) discurso 'normal' y recibir por parte del alocutario, de forma consciente o subconsciente, un juicio de adecuación en términos del grado de verosimilitud del enunciado.

En este sentido, además, el fenómeno de intertextualidad se vincula también de alguna manera con el tema del poder, en términos de su potencialidad para pautar, a través de representaciones fuertemente sancionadas socialmente, configuraciones particulares de alta estabilidad, 'orden del discurso' en sentido foucaultiano, que se constituyen en hegemónicas. A este respecto, Fairclough (1995), por ejemplo, ha mostrado que efectivamente la creatividad discursiva no está necesariamente disponible en la práctica para todos los usuarios de lenguaje, sino que se halla condicionada por diversas relaciones de poder.

\section{Metodología}

En el trabajo se analizaron un total de 25 juicios orales, de diferentes delitos, correspondientes al período de aplicación del Plan Piloto de la Reforma Procesal chilena en la IV Región. Para estos efectos, se empleó un método cualitativo de análisis de contenido, aplicándose los procedimientos de la grounded theory (Glaser \& Strauss, 1967), a través del software Atlas/ti, versión 5.2. De acuerdo con el modelo, los textos se sintetizaron en categorías conceptuales que aparecieron contextualmente y a las cuales se les asignó un código abierto (Rodríguez, Gil \& García, 1996; Strauss \& Corbin, 1998).

Posteriormente, se procedió a agrupar los códigos según sus propiedades; de esta forma se pudo obtener un conjunto de códigos que conformó una unidad superior mediante el recurso de una codificación axial, obteniéndose como resultado un modelo compuesto por relaciones generales entre los elementos, lo que permitió generar una explicación teórica desde la base.

\section{I. Propósito}

Con vistas a contribuir parcialmente en el conocimiento de que dispone el sujeto hablante para realizar adecuadamente un 'juego de lenguaje' que le permita construir un enunciado con un grado de adecuación de verosimilitud estimable, el trabajo procura establecer el rol que cumple la categoría retórica clásica de status (Quintiliano, 1916) en el entramado intertextual del discurso de los juicios orales.

Como se espera mostrar, la noción (semántica) de status cumple un papel en el sentido de generar un proceso textual que se expresa en una conformación superestructural determinada (van Dijk, I983), que a su vez refiere un tipo de representación social, en términos del 'modo' en que este tipo de discurso 'se aparece' socialmente, y que se vincula con el tema del poder en tanto en cuanto práctica social hegemónica. 


\section{Status y superestructura}

En la tradición retórica clásica, la labor de descripción del conocimiento involucrado en la conformación semántica de los discursos fue abordada parcialmente en la teoría de la narratio, por ejemplo, por medio del establecimiento de los elementa narrationis, (Lausberg, 1966), o a través del tratamiento de los topoi (Aristóteles, 1990). De forma más específica, sin embargo, no se encuentra sino a partir de Quintiliano (19|6), en la consideración de la característica discursiva de status, entendido como una propiedad semántica general común de los discursos de litigio: "/.../ id quod est commune omnibus, qui sit status /.../" (Quintiliano, 1916:326).

A pesar de que la noción clásica de status se encuentra vinculada en varios aspectos, por ejemplo, con las de 'tema' (Rastier, 2005), 'tópico' (Firbas, 1964), o 'macroestructura' (van Dijk \& Kintsch, 1983), en el sentido de constituir aquello de que trata la causa "/.../ quod in hoc causa consistat /.../" (Quintiliano, 1916: 328), por contra, no pueden hacerse del todo equivalentes, ya que mientras estas 'recorren' en sentido integrativo la totalidad del discurso, para Quintiliano (1916) el status constituye más bien una particularidad semántica característica del genus iudiciale, hecho de naturaleza propiamente relacional a partir de la configuración de la actio,y en este sentido, un concepto que se aproxima a la noción intuitiva profesional de los litigantes de los juicios orales, denominada 'teoría del caso' (Baytelman \& Duce, 2004).

El status representa, en este sentido, una suerte de 'marca textual' de la teoría del caso ".../ in hoc enim causa consistet” (Quintiliano, 1916: 330), esto es, el aspecto sobre el que los litigantes focalizan su propósito y respecto del cual deberá decidir el juez
"\%.../ quod et orator praecipue sibi obtinendum et judex spectandum maxime intellegit /.../" (Quintiliano, 1916:330).

\section{Hallazgos}

\section{I. Argumentos de la fiscalía}

Como se espera mostrar, el establecimiento del status pauta y se expresa formalmente en el discurso en términos de una conformación superestructural particular (van Dijk, 1983). El espacio dialogal argumentativo de este tipo de discursos constituye propiamente una parte del todo denominado "juicio oral', el que contiene también otros fragmentos no argumentativos ni dialogales, tales como los Antecedentes Generales, la Autoapertura, la Deliberación y la Sentencia, no contemplados en el análisis. A fin de facilitar la lectura y crítica, se presenta la superestructura de la porciones propiamente argumentativas y dialogales de forma parcelada, a través de los componentes: I. 'Argumentos presentados por la Fiscalía', II. 'Argumentos presentados por la Defensa', III. 'Alegato presentado por la Fiscalía', IV. 'Alegato presentado por la Defensa', V. 'Réplica presentada por la Fiscalía', y VI. 'Contrarréplica presentada por la Defensa', respectivamente.

La determinación del status, formalmente focalizada en el verbo (fecisti) y su intertexto (non feci), por ejemplo 'asesinó' y su intertexto implícito 'no asesinó, conforma en primer lugar el espacio discursivo superestructural: I. 'Argumentos presentados por Fiscalía', cuya representación prototípica aparece graficada en la Figura I (El paréntesis de corchete representa un camino discursivo obligatorio; el redondo, uno opcional). 


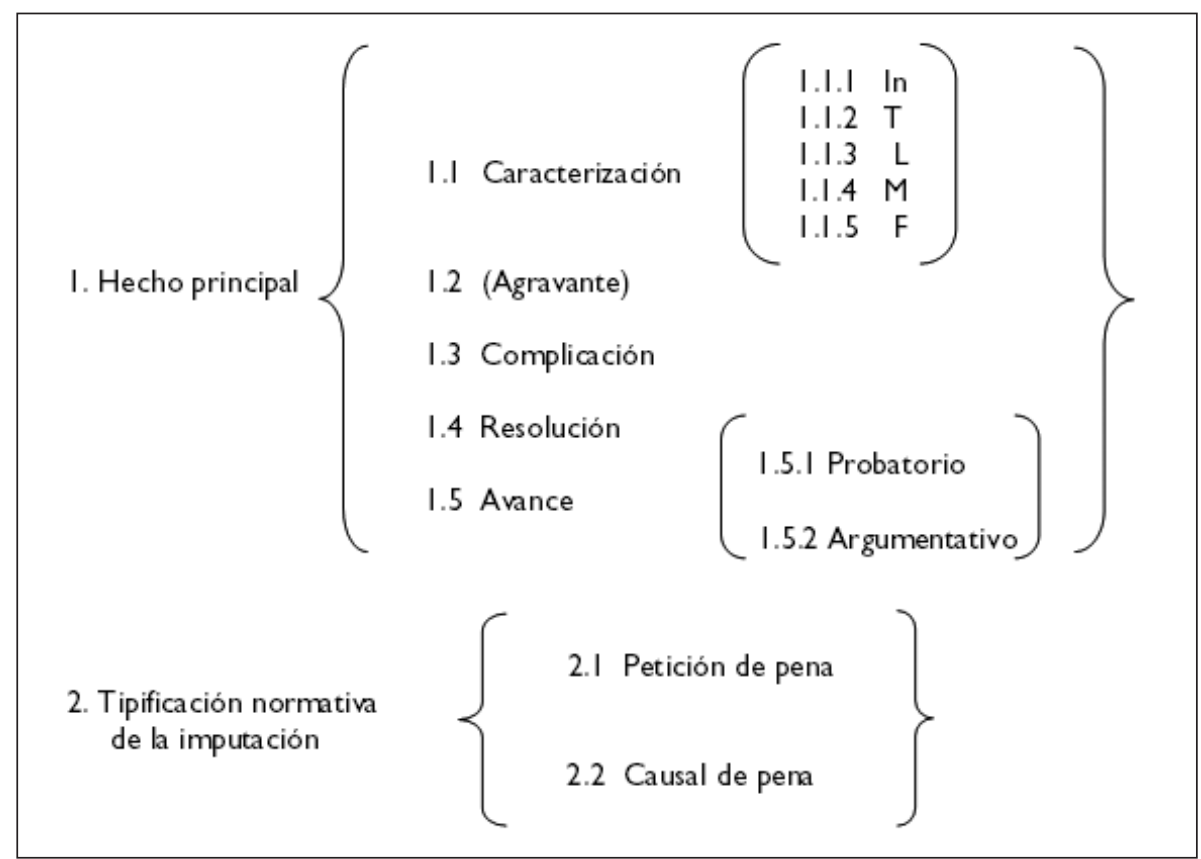

Figura I. I. 'Argumentos presentados por la Fiscalía'.

La categoría I. 'Hecho principal', representa la porción discursiva que refiere el hecho principal que ha originado la imputación. Está conformada por las subcategorías I.I 'Caracterización', porción a su vez constituida por, I.I.I 'Individualización (In)', fragmento discursivo que cumple la función de nominar, identificar y caracterizar al sujeto de la imputación; I.I.2 'Temporal (T)', porción que sitúa temporalmente la ocurrencia del Hecho principal; I.I.3 'Locativo (L)', fragmento discursivo que tiene como propósito fijar espacialmente el lugar de ocurrencia del Hecho principal; I.I.4 'Modal (M)', fragmento discursivo que refiere la forma de ocurrencia del Hecho principal; y, I.I.5 'Final (F)', porción discursiva que señala la motivación de la ocurrencia del Hecho principal.

La categoría opcional I.2 'Agravante' refiere el espacio discursivo que expresa aspectos que refuerzan la gravedad del Hecho principal. La categoría I.3 ‘Complicación' representa la porción discursiva que da cuenta de las razones que impiden que la ocurrencia del Hecho principal se desconozca pública o penalmente. La categoría I.4 'Resolución' representa el espacio discursivo que refiere el desenlace del Hecho principal, en tanto que la categoría I.5 'Avance', constituida opcionalmente por las subcategorías, I.5.I 'Avance probatorio', anuncia la presentación de pruebas sobre la ocurrencia del Hecho principal, mientras que I.5.2 'Avance argumentativo', adelanta la presentación de argumentos que garantizarían la verosimilitud de la ocurrencia del Hecho principal.

Este espacio discursivo se completa con la categoría 2. 'Tipificación normativa de la imputación', esto es, aquella porción que vincula en una relación de paratextualidad (Genette, 1992), el 'Hecho principal (I.)' con una norma determinada. Se encuentra, a su vez, conformado por las subcategorías 2.1 'Petición de pena', aquel espacio discursivo que, en virtud de la tipificación normativa, solicita se aplique la norma legal correspondiente; y 2.2 'Causal de pena', porción discursiva que refuerza la Petición de pena.

\subsection{Argumentos de la defensa}

El planteamiento de status efectuado por la fiscalía funciona como cotexto (Hanks, 2000), para generar por parte de la defensa el espacio discursivo 'll. Argumentos presentados por la Defensa', a partir de una relación de contradicción (Figura 2: La línea de doble punta representa una relación intertextual de contradicción. La simple, de asociación), entre las categorías I.I. 'Hecho principal' y II.3. 'Negación del Hecho principal'.

El status constituye per se una categoría generativa discursiva de relación intertextual en el sentido de que el defensor, a partir de la teoría del caso del fiscal " $/ . . /$ concedamus ex depulsione nasci statum I.../" (Quintiliano, 1916:332), establece por negación 


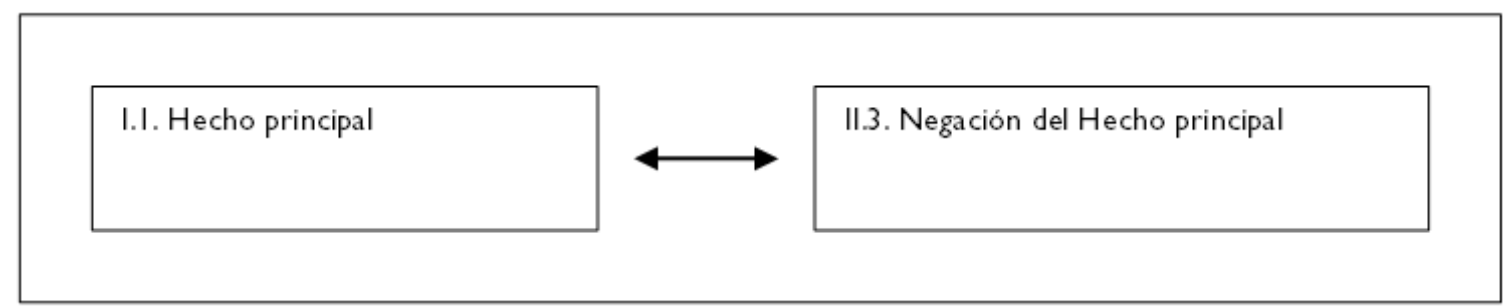

Figura 2. Relación de contradicción de cotexto.

un determinado grado del mismo, teniendo a su vez en consideración una amplia constelación de otros textos, en los que se encuentran por cierto los normativos, que contribuirían a una posible objeción o aprobación de su planteamiento de la causa. Quintiliano (1916), por su parte, considera status esenciales el de "conjetura, el de cualidad, y el de definición" "I.../ Secundum plurimos auctores servabam tres rationales status: conjecturam, qualitatem, finitionem, unum legalem. Hi mihi status generales erant" (Quintiliano, 1916: 354).

Como se dijo, la decisión semántica que supone la adecuación del grado del status por parte de la defensa, que en términos formales se localiza en las 'marcas' verbo, complemento directo y adverbio, respectivamente, contribuye consecutivamente a conformar la organización superestructural del espacio discursivo II. 'Argumentos presentados por la Defensa', en la forma que lo refiere la Figura 3.

La categoría I. 'Recuperación temática' constituye aquella porción discursiva en la que se hace referencia explícita mediante relación cotextual de la porción discursiva I. 'Argumentos presentados por la Fiscalía'. La categoría 2. 'Individualización', constituye el fragmento discursivo a través del cual se nomina, identifica y caracteriza al sujeto de la imputación.

La categoría 3. 'Negación del Hecho principal' constituye el punto central de este espacio discursivo que, como se dijo, establece una relación cotextual de contradicción entre fragmentos superestructurales

\section{Recuperación temática \\ 2. Individualización \\ 3. Negación del Hecho principal}
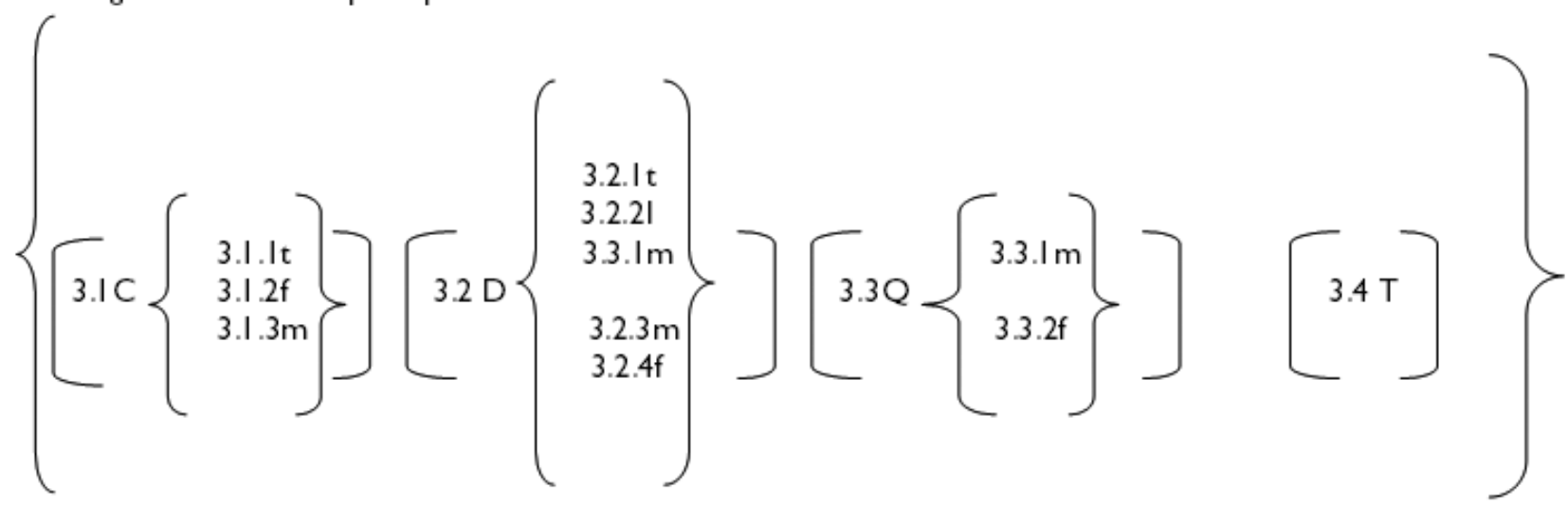

4. Tipificación normativa de $\mathrm{N}$ del Hecho principal

5. Petición de Negación de solicitud de pena

Figura 3. II. 'Argumentos presentados por la Defensa'. 
específicos. Esta porción discursiva está, a su vez, constituida por las subcategorías:

3. IC Negación por conjetura. Ante la afirmación de fiscalía 'feci', se opone la de la defensa 'non feci', de forma que surge el grado y relación intertextual de conjetura 'an fecerit?'; i.e, 'mató', 'no mató', '‘habrá matado?' Representa, en consecuencia, el espacio discursivo que niega por conjetura la Tipificación normativa de la imputación (I.2.). Puede presentar las siguientes variantes:

3.I.It Negación conjetura temporal, porción discursiva que conjetura la Tipificación normativa de la imputación por falta de correspondencia temporal en relación con la ocurrencia del Hecho principal.

3.I.2f Negación conjetura final, espacio discursivo que conjetura la Tipificación normativa de la imputación por falta de correspondencia respecto de la motivación de la ocurrencia del Hecho principal.

3.I.3m Negación conjetura modal, porción discursiva que conjetura la Tipificación normativa de la imputación por falta de correspondencia en relación con la forma de ocurrencia del Hecho principal.

3.2D Negación por definición. Ante la afirmación de fiscalía 'hoc fecisti', se opone la de la defensa 'non hoc feci', de forma que surge el grado y relación intertextual de definición quid fecerit?; i.e., 'mató', 'no mató', '¿qué hizo?: se defendió.' Constituye, por tanto, la porción discursiva que niega por definición la Tipificación normativa de la imputación. Puede presenta las siguientes variantes:

3.2.It Negación definición temporal, fragmento discursivo que redefine la Tipificación normativa de la imputación por falta de correspondencia temporal respecto de la ocurrencia del Hecho principal.

3.2.2I Negación definición locativa, espacio discursivo que redefine la Tipificación normativa de la imputación por falta de correspondencia espacial con relación a la ocurrencia del Hecho principal.

3.2.3m Negación definición modal, porción discursiva que redefine la Tipificación normativa de la imputación por falta de correspondencia de la forma de ocurrencia del Hecho principal.

3.2.4f Negación definición final, espacio discursivo que redefine la Tipificación normativa de la imputación por falta de correspondencia respecto de la motivación de ocurrencia del Hecho principal.

3.3Q Negación por cualidad. Ante la aceptación por parte de la defensa del punto central de la acusación 'etiam si feci,' introduce un grado y relación intertextual de cualidad 'recti feci'; i.e., 'efectivamente mató, pero en defensa propia'. Representa, en consecuencia, el espacio discursivo que atenúa la Tipificación normativa de la imputación. Se manifiesta a través de los siguientes subtipos:

3.3. Im Negación cualidad modal, porción discursiva que atenúa la Tipificación normativa de la imputación por falta de correspondencia de la forma de ocurrencia del Hecho principal.

3.3.2f Negación cualidad final, fragmento discursivo que atenúa la Tipificación normativa de la imputación por falta de correspondencia de la motivación de ocurrencia del Hecho principal.

3.4T Negación por traslación. La estrategia constituye un 'cambio de causa'; i.e., introducir otro punto de disputa (translationis). Constituye, por ello, la porción discursiva que modifica el tema de discusión del Hecho principal.

La categoría 4. 'Tipificación normativa de $\mathrm{N}$ (la negación) del Hecho principal' representa aquella porción discursiva que, a través de una relación de paratextualidad, vincula el Hecho principal (I.I.) con una norma diferente a la referida en el espacio superestructural I. 'Argumentos presentados por la Fiscalía'.

La categoría 5. 'Petición de Negación de solicitud de pena' constituye aquella porción discursiva que, a través de una constelación relacional, intertextual, paratextual, cotextual, metatextual (Hanks, 2000), entre otras, solicita absolución de la imputación.

\subsection{Alegato de la fiscalía. Alegato de la defensa}

El establecimiento del grado de status por parte de la defensa da pie cotextual para generar por parte de la fiscalía el espacio superestructural III. 'Alegato presentado por la Fiscalía', a partir de una relación intertextual tanto de contradicción (Figura 4), entre la categorías II.3. 'Negación del Hecho principal' y III.I. 'Refutación a la defensa', como de asociación con la categoría I.I. 'Hecho principal'.

La decisión que supone el establecimiento del grado de status por parte de la defensa pauta la organización superestructural del consecuente espacio discursivo, en la forma que lo refiere la Figura 5. 


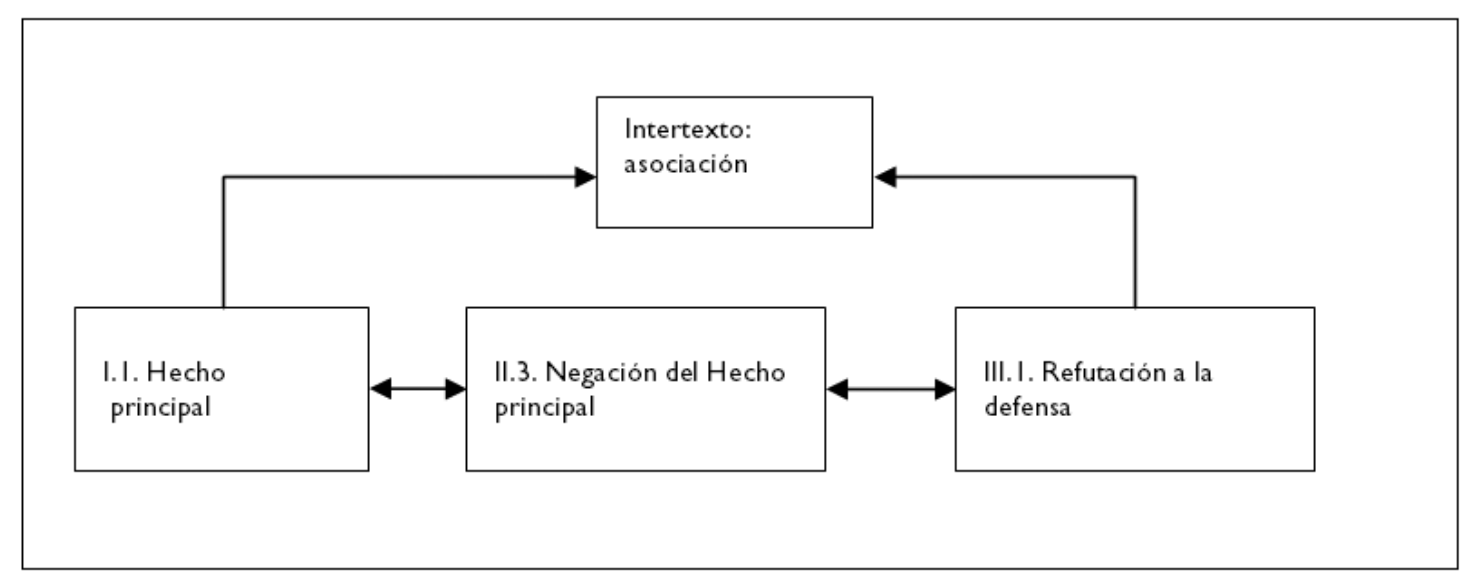

Figura 4. Relación cotextual de contradicción y de asociación.

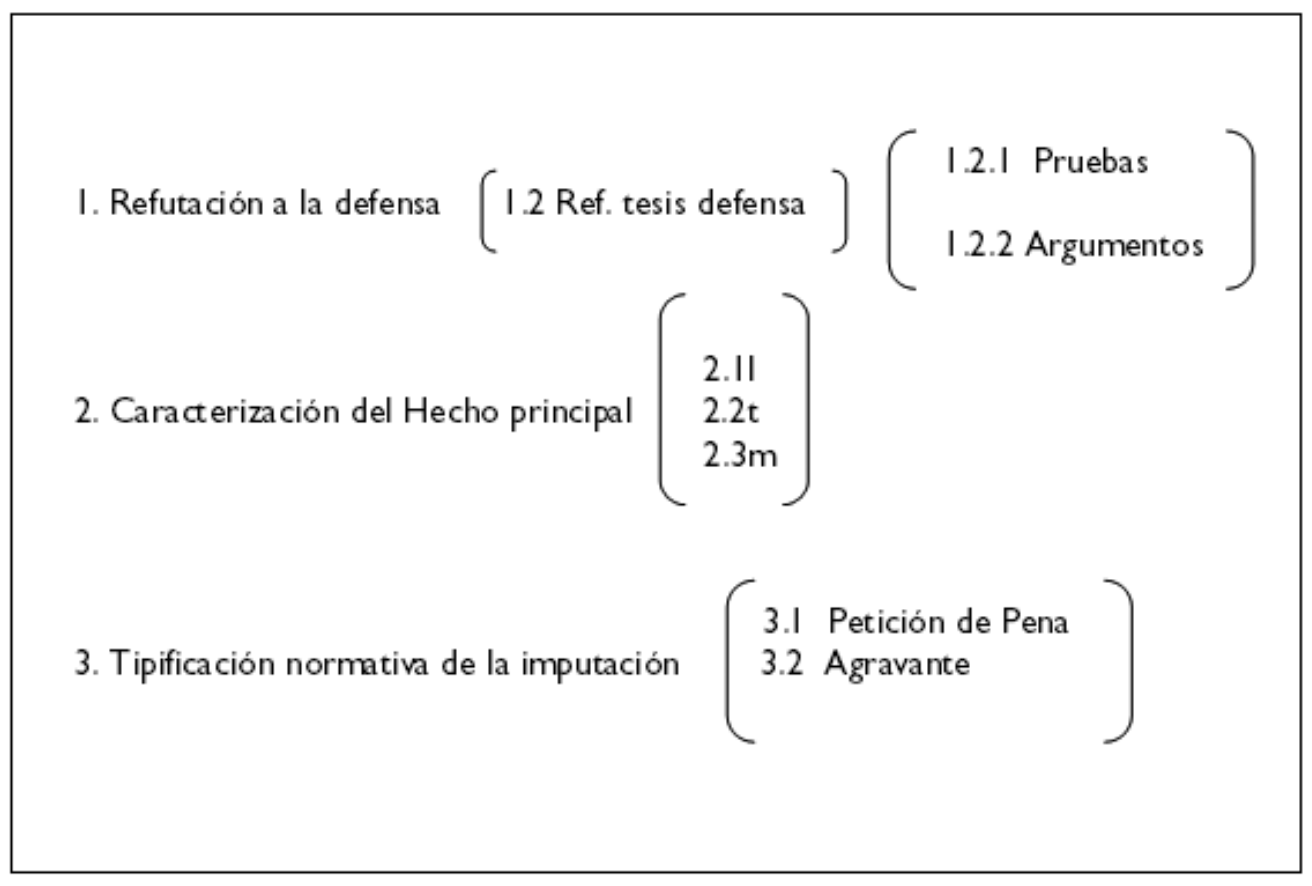

Figura 5. III. 'Alegato presentado por la Fiscalía'.

La categoría I. 'Refutación de la defensa' constituye aquella porción discursiva que, en relación metatextual explícita, rechaza la Negación del Hecho principal (II.3.), a través de las subcategorías: I.2 'Referencia (Ref.) tesis de la defensa', fragmento discursivo que recupera y analiza el status referido en la porción discursiva Negación del Hecho principal; I.2.I 'Pruebas', fragmento discursivo que acredita la ocurrencia del Hecho principal y, I.2.2 'Argumentos', porción discursiva que refuerza la verosimilitud de la ocurrencia del Hecho principal.
La categoría 2. 'Caracterización del Hecho principal' representa la porción discursiva que reafirma intertextualmente la porción discursiva Hecho principal (I.I.), a través de las subcategorías, 2.II 'Locativo', a saber, fragmento discursivo que reafirma espacialmente la ocurrencia del Hecho principal; $2.2 t$ 'Temporal', porción discursiva que reafirma temporalmente la ocurrencia del Hecho principal y $2.3 \mathrm{~m}$ 'Modal', fragmento discursivo que reafirma la forma de ocurrencia del Hecho principal. 


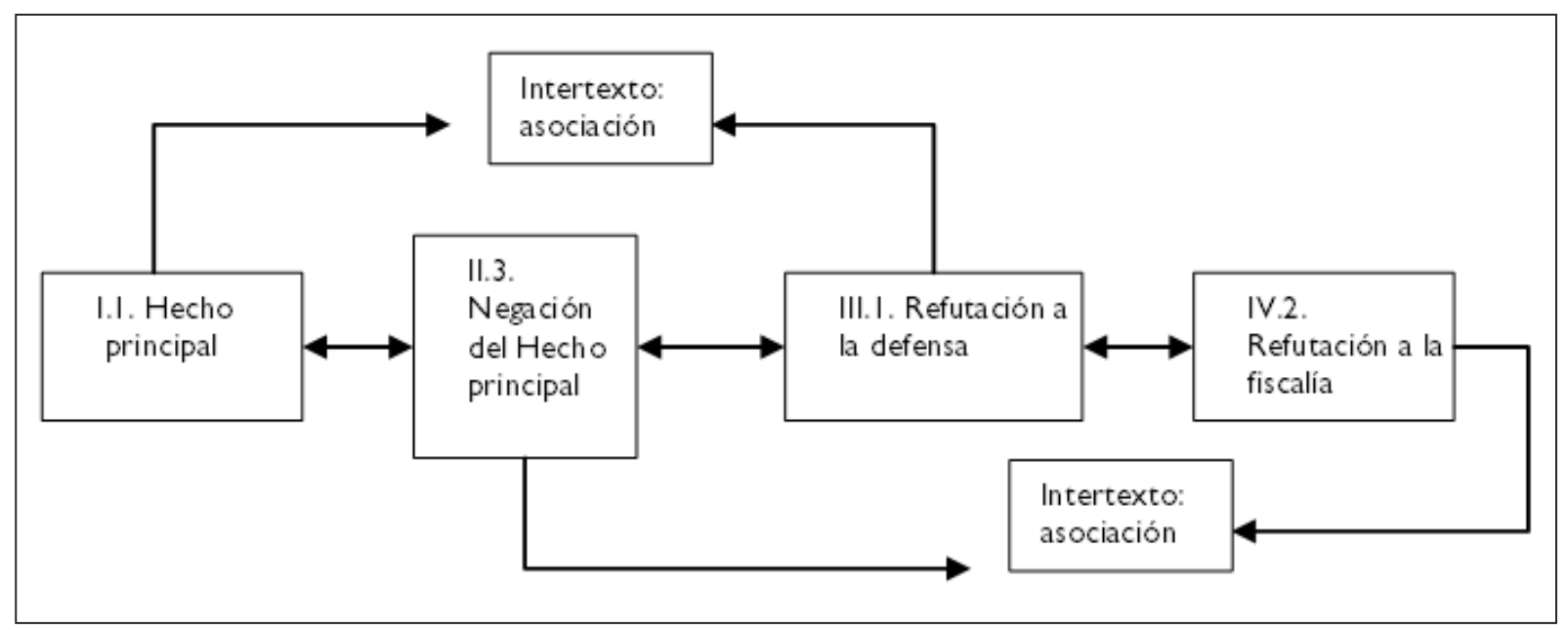

Figura 6. Relación múltiple cotextual de contradicción y de asociación.

La categoría 3. 'Tipificación normativa de la imputación', por su parte, constituye el espacio discursivo que refuerza la categoría Tipificación normativa de la imputación (I.2.), referida en la porción superestructural l.'Argumentos presentados por la Fiscalía', a través de la subcategorías 3.I 'Petición de pena', porción discursiva que refuerza intertextualmente la porción discursiva 2.I 'Petición de pena', y 3.2 'Agravante', espacio discursivo que refuerza intertextualmente la porción discursiva 1.2 (Agravante).

El juego de lenguaje que supone la dialéctica del status genera sucesivas relaciones intertextuales pautando la generación del espacio superestructural IV. 'Alegato presentado por la Defensa', en el que se amplía la relación de contradicción entre las categorías IV.2. 'Refutación a la fiscalía', III.I. 'Refutación a la defensa', y I.I. 'Hecho principal'. En tanto, se multiplica la relación de asociación entre las categorías IV.2. 'Refutación a la fiscalía' y II.3. 'Negación del Hecho principal', según muestra la Figura 6.

La configuración superestructural de esta nueva porción discursiva (IV.) se expresa en la forma que refiere la Figura 7.

La categoría I.'Recuperación temática' es expresión directa de aquella relación intertextual que retoma la porción III. 'Alegato presentado por la Fiscalía'.

La categoría 2. 'Refutación a la fiscalía' representa la porción discursiva que establece relación intertextual

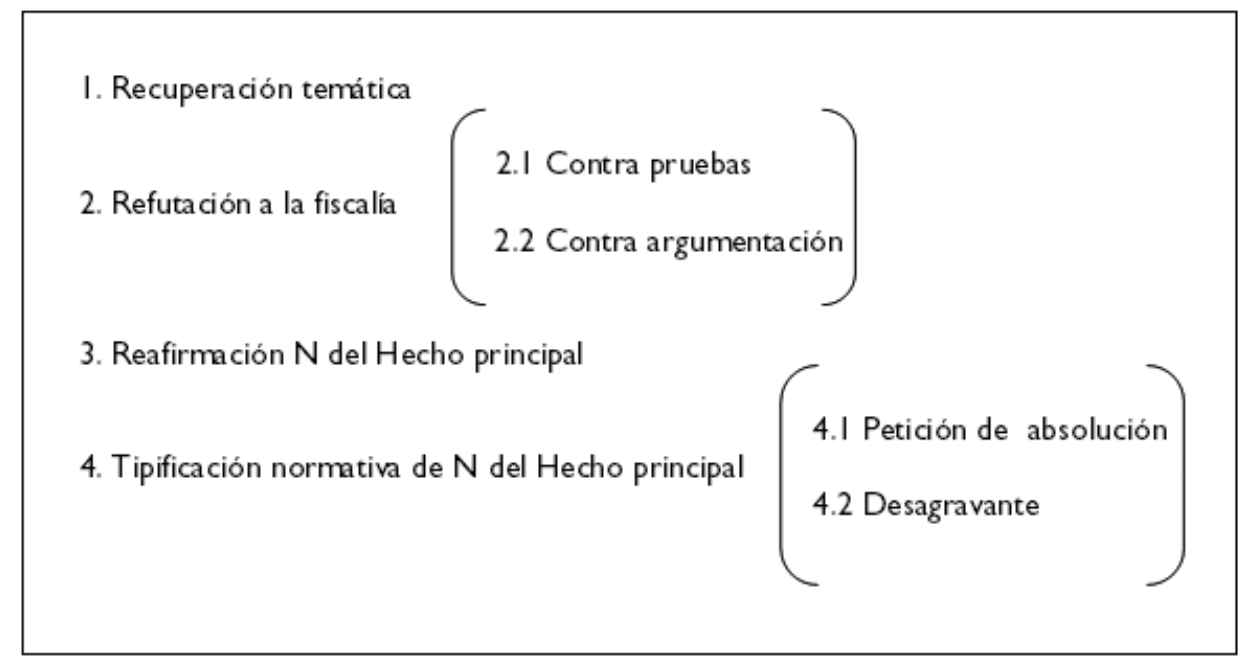

Figura 7. IV. 'Alegato presentado por la Defensa'. 
de contradicción con el espacio superestructural III. 'Alegato presentado por la Fiscalía', a través de las subcategorías 2.I 'Contra pruebas', espacio discursivo que rechaza el fragmento discursivo III.I.2.I 'Pruebas', y 2.2 'Contra argumentación', porción discursiva que rechaza el espacio discursivo III.I.2.2 'Argumentos'.

La categoría 3. 'Reafirmación de $\mathrm{N}$ (negación) del Hecho principal' representa aquel espacio discursivo que establece una relación intertextual de asociación que reafirma la porción textual II.3. 'Negación del Hecho principal'.

La categoría 4. 'Tipificación normativa de $\mathrm{N}$ (negación) del Hecho principal' establece, a su vez, una relación intertextual de asociación con la porción discursiva II.4. 'Tipificación normativa de $\mathrm{N}$ (negación) del Hecho principal'. Las categorías 4.I 'Petición de absolución' y 4.2 'Desagravante', por su parte, constituyen los fragmentos discursivos que establecen relación intertextual de asociación con la categoría II. 5. 'Petición de Negación de solicitud de pena'.

\subsection{Réplica de la fiscalía}

Las múltiples relaciones intertextuales generadas a partir de la relación de status contribuyen a conformar la siguiente porción superestructural en la que se multiplica la relación de contradicción y asociación, particularmente a partir de la categoría V.I.'Refutación de N (negación) del Hecho principal', según lo muestra la Figura 8.

Las categorías que resultan ser expresión de estas relaciones múltiples se manifiestan en la porción superestructural V. 'Réplica presentada por la Fiscalía', en la forma que muestra la Figura 9.

La categoría I. 'Refutación N (negación) del Hecho principal' constituye la porción discursiva que rechaza la porción textual IV. 'Alegato presentado por la Defensa', a través de las subcategorías I.I 'Contra pruebas', fragmento discursivo que rechaza la porción discursiva IV.2.2.I 'Contra pruebas'; y I.2 'Contra argumentación', fragmento discursivo que rechaza la porción discursiva IV.2.2.2 'Contra

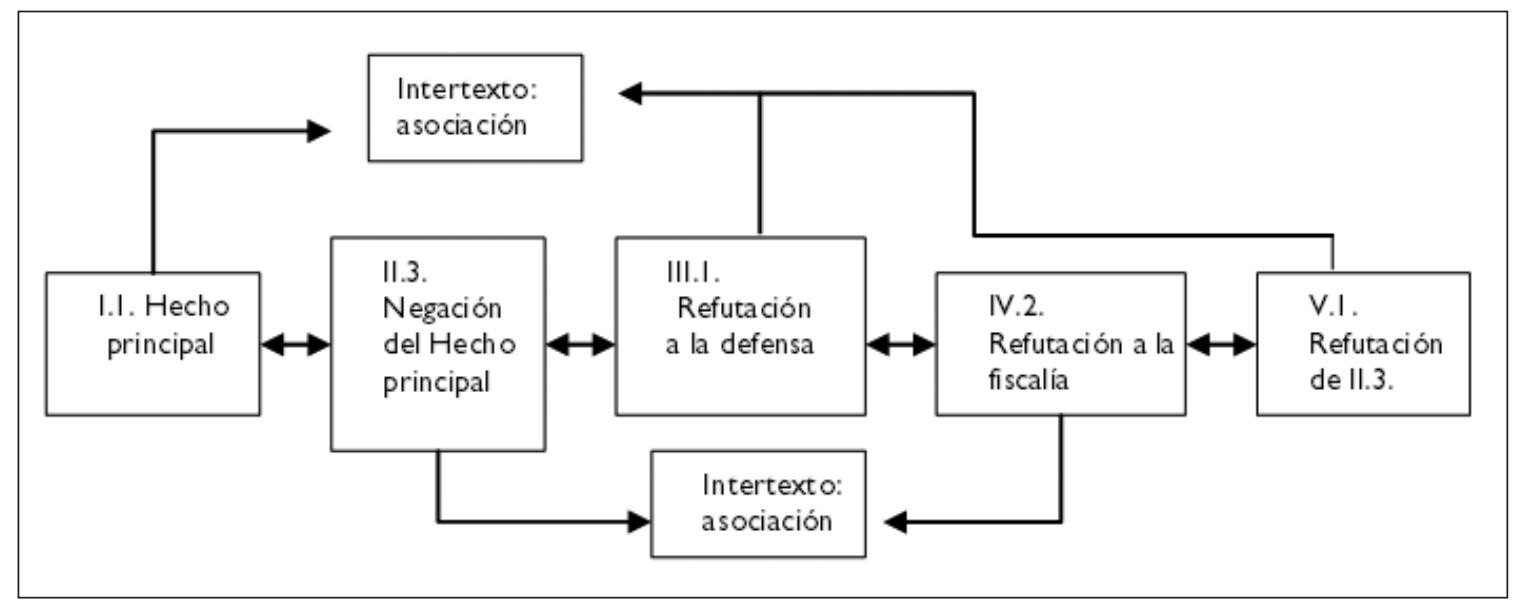

Figura 8. Relación múltiple cotextual de contradicción y de asociación.

I. Refutación $\mathrm{N}$ del Hecho principal
2. Reafirmación del Hecho principal
3. Tipificación normativa de la imputación

Figura 9. V. 'Réplica presentada por la Fiscalía’. 
argumentación’. La categoría opcional I.3 ‘Nuevas pruebas', por su parte, reafirma la porción discursiva I.I.'Hecho principal'.

La categoría 2. 'Reafirmación del Hecho principal' representa aquella porción discursiva que refuerza intertextualmente la porción I. 'Hecho principal'. La categoría 3. 'Tipificación normativa de la imputación' constituye la categoría que establece la relación intertextual que refuerza la categoría 2. 'Tipificación normativa de la imputación', referida en la porción discursiva l.'Argumentos presentados por la Fiscalía' y/o en la porción textual III. 'Alegato presentado por la Fiscalía'.

\subsection{Contrarréplica de la defensa}

La dinámica discursiva que genera la relación de status se resuelve finalmente en la última porción superestructural, espacio que amplía las relaciones intertextuales de asociación y contradicción, particularmente a partir de la categoría VI.2.
'Tipificación normativa de N (negación) del Hecho principal', según muestra la Figura 10.

Las relaciones gatilladas por la tensión del status, finalmente, contribuyen a conformar el último espacio superestructural de este tipo de discurso, la VI. 'Contrarréplica presentada por la Defensa', según lo refiere la Figura II.

La categoría I. 'Referencia a Refutación N (de la negación) del Hecho principal' representa aquella porción discursiva que rechaza el espacio discursivo V.I.'Refutación de N (negación) del Hecho principal', por medio de la subcategorías, I.I 'Contra pruebas', fragmento discursivo que rechaza la acreditación de la porción textual V.I.I.I 'Contra pruebas'; I.2 'Contra argumentación', porción discursiva que rechaza la verosimilitud de la porción textual V.I.I.2 'Contra argumentación', y I.3 'Nuevas pruebas', porción discursiva opcional que acredita la categoría II.3. 'Negación del Hecho principal'.

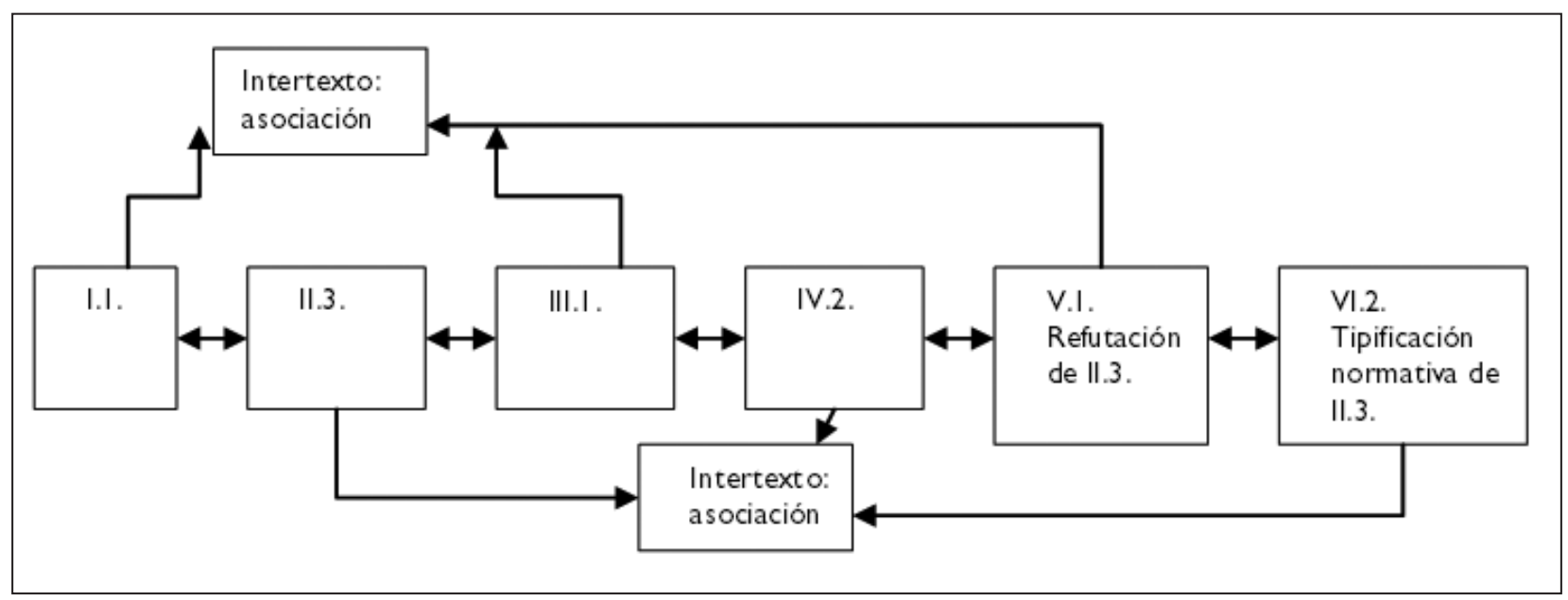

Figura 10. Relaciones cotextuales de contradicción y asociación.

$\left.\begin{array}{|l}\text { I. Referencia a Refutación } \mathrm{N} \text { del Hecho principal } \\ \text { 2. Tipificación normativa de } \mathrm{N} \text { del Hecho principal }\end{array}\right)$

Figura I I. VI. 'Contrarréplica presentada por la Defensa'. 
La categoría 2. 'Tipificación normativa de $\mathrm{N}$ (negación) del Hecho principal' constituye el fragmento discursivo que refuerza la Tipificación normativa de la imputación referida en la porción textual II. 'Argumentos presentados por la Defensa' y/o en la porción textual IV. 'Alegato presentado por la Defensa'. La categoría 2.I 'Petición de absolución', finalmente, constituye la porción discursiva que reafirma el fragmento discursivo II.5. 'Petición de Negación de solicitud de pena', y/o IV.4.4.I 'Petición de absolución'.

\section{CONCLUSIONES}

Como se esbozó, la naturaleza dialéctica de la práctica discursiva que se ha instaurado a partir de los discursos representados por los juicios orales supone una objeción implícita al racionalismo propio de la lógica formal en cuanto mecanismo de prueba y asume que una supuesta verdad puede emanar de un ejercicio verbal de contraste de pareceres diversos.

La determinación de las estrategias formales que emplean los participantes de esta modalidad discursiva representa para la teoría lingüística una tarea ética ineludible, en el sentido de que una construcción verbal determinada podría ser razón, sin más, para que un sujeto involucrado en una causa modifique, por ejemplo, su condición de imputado a la de condenado.

La metodología seguida en el trabajo posibilitó tratar un corpus de 25 juicios orales, a partir del cual, luego de que la saturación provista por el análisis permitiera obtener categorías contextuales agrupadas según sus propiedades mediante el recurso de una codificación axial, se pudo obtener como resultado un modelo de superestructura del juicio oral, generado teóricamente desde 'la base'.

Según se pretendió mostrar, la noción de status, tratada preferentemente en el marco de la doctrina retórica clásica, cumple un rol generativo discursivo, en el sentido de pautar un proceso textual que se expresa en una conformación superestructural determinada. En este sentido, el trabajo ha pretendido aportar parcialmente al conocimiento de la relación existente entre contenido y forma.

Si bien próxima a categorías semánticas como las de tema o macroestructura, la noción de status constituye un fenómeno propiamente relacional, aparentemente característico del genus iudiciale clásico, y por tanto, una categoría respecto de la cual habría que profundizar a través de más investigación, en atención a que parece traspasable también hacia otros tipos de géneros dialógicos, en tanto en cuanto la depulsio quintiliana no es necesariamente exclusiva de este género en particular.

En su condición de fenómeno relacional, la noción de status no constituye propiamente un 'objeto' de análisis en sentido usual, ya que no se expresa sino a través de categorías generales como la superestructura, o la microestructura, dependiendo del grado que adquiera en un discurso específico.

Dada su condición de contribuir a la conformación superestructural del discurso, la noción de status refiere, a la vez, cierto tipo de representación social, en la forma prototípica de una práctica discursiva determinada, que puede llegar a convertirse en expresión de poder dada su potencialidad de constituir forma discursiva hegemónica, un orden del discurso capaz de pautar conductas específicas: sentencias (des) favorables, con atención del grado de verosimilitud alcanzado por la forma del enunciado.

Aun cuando el trabajo permitió, principalmente por razones metodológicas, establecer algunos tipos de ellas, no ha sido pretensión del mismo establecer el haz de relaciones intertextuales que surgen a partir de la noción de status, sino solo determinar cómo estas se expresan en la conformación superestructural del juicio oral. Cabe pues, indagar a partir de esta categoría la ley del infinito de sus incrementos. 


\section{REFERENCIAS BIBLIOGRÁFICAS}

Aarnio,A. \& MacCormick, N. (1992). Legal reasoning: New York: New York University Press.

Adams, E. (1 983). Probabilistics enthymemes. Journal of Pragmatics, 7, 283-295.

Albaladejo, T. (1993). Retórica. Madrid: Síntesis.

Alexy, R.( ( 989). Teoría de la argumentación jurídica.Madrid: Centro de Estudios Constitucionales.

Aristóteles (1990). Retórica. Madrid: Centro de Estudios Constitucionales.

Atienza, M. (1993). Las razones del derecho. Madrid: Centro de Estudios Constitucionales.

Barthes, R. (1974). Investigaciones retóricas I. La antigua retórica. Ayudamemoria. Buenos Aires: Editorial Tiempo Contemporáneo.

Barthes, R. (1973). Le plaisir du texte. París: Seuil.

Baytelman,A. \& Duce, M. (2004). Manual de litigación en juicios orales. Santiago de Chile: Centro de Estudios de Justicia de Las Américas.

Campagna, M. \& Lazzaretti, A. (1998). Lógica, argumentación y retórica. Buenos Aires: Biblios.

Cicerón, M. (199I). Retórica a Herenio. Barcelona: Bosch.

De Beaugrande, R. \& Dressler,W. (I 98I). Introduction to text linguistics. Londres: Longman.

Fairclough, N. (1995). Critical discourse analysis. London: Longman.

Farr, R. ( 1 988). Las representaciones sociales. Psicología Social II, 495-506.

Feteris, E. (1997). A survey of 25 years of research on legal argumentation. Argumentation, I I (2), 355-376.

Firbas J. (1964). On defining the theme in functional sentence analysis. Travaux Linguistiques de Prague, I, 267-280.

Glaser, B. \& Strauss,A. (1967). The discovery of grounded theory: Strategies for qualitative research. New York:Aldine de Gruyter.

Genette, G. (1992). The architext:An introduction. Berkeley: University of California Press.

González , J. (1988). Tratado histórico de retórica filosófica. Madrid: Nájera.

Hanks, W. (2000). Intertexts writings on language, utterance, and context. Lanham, Maryland: Rowman \& Littlefield Publishers.

Kristeva, J. (1967). Bakhtine, le mot, le dialogue et le roman. Critique, 239, 440-44I.

Laborda, X. (1996). Retórica interpersonal. Barcelona: Octaedro.

Lausberg, H. (1996). Manual de retórica literaria. Madrid: Gredos.

Martín, L. (1998). Poder-decir o el poder de los discursos. Madrid: Arrecife.

Mayoral, J. ( 1994). Figuras retóricas. Madrid: Editorial Síntesis.

Murphy, J. (1989). Sinopsis histórica de la retórica clásica. Madrid: Gredos.

Noemi, C. (1999). Estructura social y contenido del discurso. Pragmalingüística, 7, I29-I 37.

Perelman Ch. \& Olbrechts-Tyteca L. (1989). Tratado de la argumentación. La nueva retórica. Madrid: Gredos.

Quintiliano, M. (1916). Instituciones oratorias. Madrid: Librería de Perlado y Páez.

Rastier, F. (2005). Semántica interpretativa. México, D.F.: Siglo XXI. 
Rivano, J. (1984). Lógica práctica y lógica teórica. Lund: Ms. Universidad de Lund.

Rodríguez, G., Gil J. \& García E. (1996). Metodología de la investigación cualitativa. Málaga:Aljibe.

Strauss, A. \& Corbin, J. (1998). Basics of qualitative research. Techniques and procedures for developing grounded theory. London: Sage.

Toulmin, S., Rieke, R. \& Janik, A. (1979). An introduction to reasoning. New York: Mcmillan.

Walton, D. (1996). A pragmatic theory of fallacy. Tuscaloosa: University of Alabama Press.

van Dijk, T. (1983). La ciencia del texto. Barcelona: Paidós.

van Dijk, T. (200I). El discurso como estructura y proceso. Barcelona: Gedisa.

van Dijk, T. \& Kintsch, W. (1983). Strategies of discourse comprehension. New York: Academic Press.

Van Eemeren, F., Grootendorst, R. \& Kruiger, T. (1987). A handbook of argumentation theory. Holanda/U.S.A: Foris Publications.

* Este trabajo se enmarca en el proyecto de investigación PRO8204 'Estrategias argumentales utilizadas en los primeros juicios orales de la IV Región Coquimbo: Levantamiento de una teoría fundamentada acerca del discurso jurídico', financiado por la Dirección de Investigación de la Universidad de La Serena. 